\title{
The effect of liquid fibre on gastric emptying in the rat and humans and the distribution of small intestinal contents in the rat
}

\author{
J Tomlin, N Brown, A Ellis, A Carlsson, C Bogentoft, N W Read
}

\begin{abstract}
A combination of the polysaccharide ethylhydroxyethylcellulose (EHEC) and the surfactant sodiumdodecylsulphate (SDS) has the extraordinary physical property of being liquid at room temperature but gelling firmly at $37^{\circ} \mathrm{C}$. It has been named 'liquid fibre' and its effect on gastric emptying has been tested in rats and humans, as well as its effect on intestinal distribution in rats. Rats were gavaged with $5 \mathrm{ml}$ of radiolabelled liquid fibre, SDS in water, or water control. Subgroups were killed after $25,50,100,200$, and 300 minutes, the gut removed, and the distribution of radioactivity measured scintigraphically. Liquid fibre gelled in the stomach and spread exponentially down the small intestine before 25 minutes. This distribution was maintained for $\mathbf{2 0 0}$ minutes after which the stomach began to empty again. In the human study, 10 healthy men drank $250 \mathrm{ml}$ liquid fibre and placebo labelled with $1.85 \mathrm{MBq}$ technetium tin colloid on separate occasions. Gastric emptying was measured by gammacamera. Half emptying time significantly increased from $17 \cdot 7$ to $55 \cdot 8$ minutes (means, $p<0.05$ ). The time for $10 \%$ to empty (which includes any lag time) increased from 7.0 to 19.4 minutes $(p<0.05)$. Average emptying rate decreased from $4.49 \% / \mathrm{min}$ for placebo to $1 \cdot 60 \% / \mathrm{min}$ for liquid fibre $(\mathrm{p}<0.01)$. The dramatic delay in gastric emptying suggests liquid fibre may have clinical applications while its liquid formulation should improve acceptability.

(Gut 1993; 34: 1177-1181)
\end{abstract}

The combination of the polysaccharide called ethylhydroxyethylcellulose (EHEC) and the surfactant, sodiumdodecylsulphate (SDS) has the extraordinary physical property of being a flowing liquid at room temperature but setting into a firm gel at $37^{\circ} \mathrm{C}$. The mixture has been called liquid fibre because the polysaccharide it contains is a chemically modified cellulose derived from cotton plants, and is therefore included in the definition of 'dietary fibre' (any substance of plant origin which is undigested by human alimentary enzymes).' Table I shows its composition.

The formation of a gel at body temperature should mean that after ingestion, liquid fibre would be expected to delay the emptying of gastric contents. Slowing the rate of gastric emptying may be useful in the treatment of dumping syndrome, to reduce the rate of glucose absorption in some patients with diabetes, and as a stratagem to reduce food intake. Liquid fibre also has bioadhesive properties and so may prove useful for targeting drug delivery in the gastrointestinal tract.

These possible benefits are dependent on the gel structure being maintained in the gut and resisting disruption by gastric secretions and motility. In vitro studies have shown that the gel is maintained in the presence of simulated gastric juice $(3.2 \mathrm{~g}$ pepsin in $7 \mathrm{ml}$ hydrochloric acid mixed with $2 \mathrm{~g} \mathrm{NaCl}$ and $1 \mathrm{l}$ water), but in vivo studies are needed to establish liquid fibre's properties and effects. We have therefore tested the effects of liquid fibre on gastric emptying in rats and humans and the distribution in the small intestine of rats.

\section{Methods}

\section{RAT STUDY}

A total of 79 albino rats were allocated to one of three groups; test (EHEC/SDS), SDS control, and water control. The EHEC/SDS mixture was prepared by dispersing EHEC in sterile water ( $1 \% \mathrm{wt} / \mathrm{vol}$ ), refrigerating overnight to improve solubility, and then adding $4 \mathrm{mM}$ SDS. The SDS control solution was prepared by dissolving $4 \mathrm{mM}$ SDS in sterile water. Each rat was gavaged with $5 \mathrm{ml}$ of the appropriate solution into which a known amount of technetium- $99 \mathrm{~m}\left({ }^{99 \mathrm{~m}} \mathrm{Tc}\right)$ tin colloid (Amersham, Bucks, UK) had been mixed so that each rat received approximately $1 \mathrm{MBq}$ per $5 \mathrm{ml}$. The presence of the radioactive colloid did not affect the gelling properties. The three groups of rats were further divided into subgroups of five rats that were killed 25, 50, 100, 200 , and 300 minutes after gavage by exposure to cyclopropane gas. Two additional rats were included in the 50 minutes water control $(n=7)$ and one additional rat was included in both the 100 minutes and 200 minutes water controls $(n=6)$.

TABLE I Composition of liquid fibre and placebo used in the human studies

\begin{tabular}{lcc}
\hline & \multicolumn{2}{c}{ Mass in $100 \mathrm{~g}$} \\
\cline { 2 - 3 } Component & Liquid fibre & Placebo \\
\hline Ethylhydroxyethylcellulose (EHEC) & $850 \mathrm{mg}$ & - \\
Sodiumdodecylsulphate & $87 \mathrm{mg}$ & - \\
Methyl-p-hydroxybenzoate & $50 \mathrm{mg}$ & $50 \mathrm{mg}$ \\
Sorbic acid & $150 \mathrm{mg}$ & $150 \mathrm{mg}$ \\
PFW Pomerans aroma & $1 \mathrm{~g}$ & $1 \mathrm{~g}$ \\
Glycerol & $2.6 \mathrm{~g}$ & $2.6 \mathrm{~g}$ \\
Purified water, up to & $100 \mathrm{~g}$ & $100 \mathrm{~g}$ \\
\hline
\end{tabular}

Manufactured according to GMP by C Eriksson-Lind at Kab Pharma, Solna, Sweden (batch numbers AOL002 and AOL003, respectively). EHEC of medical grade was obtained from Berol Nobel AB, Stenungsund, Sweden. Liquid fibre has been patented in Europe. 

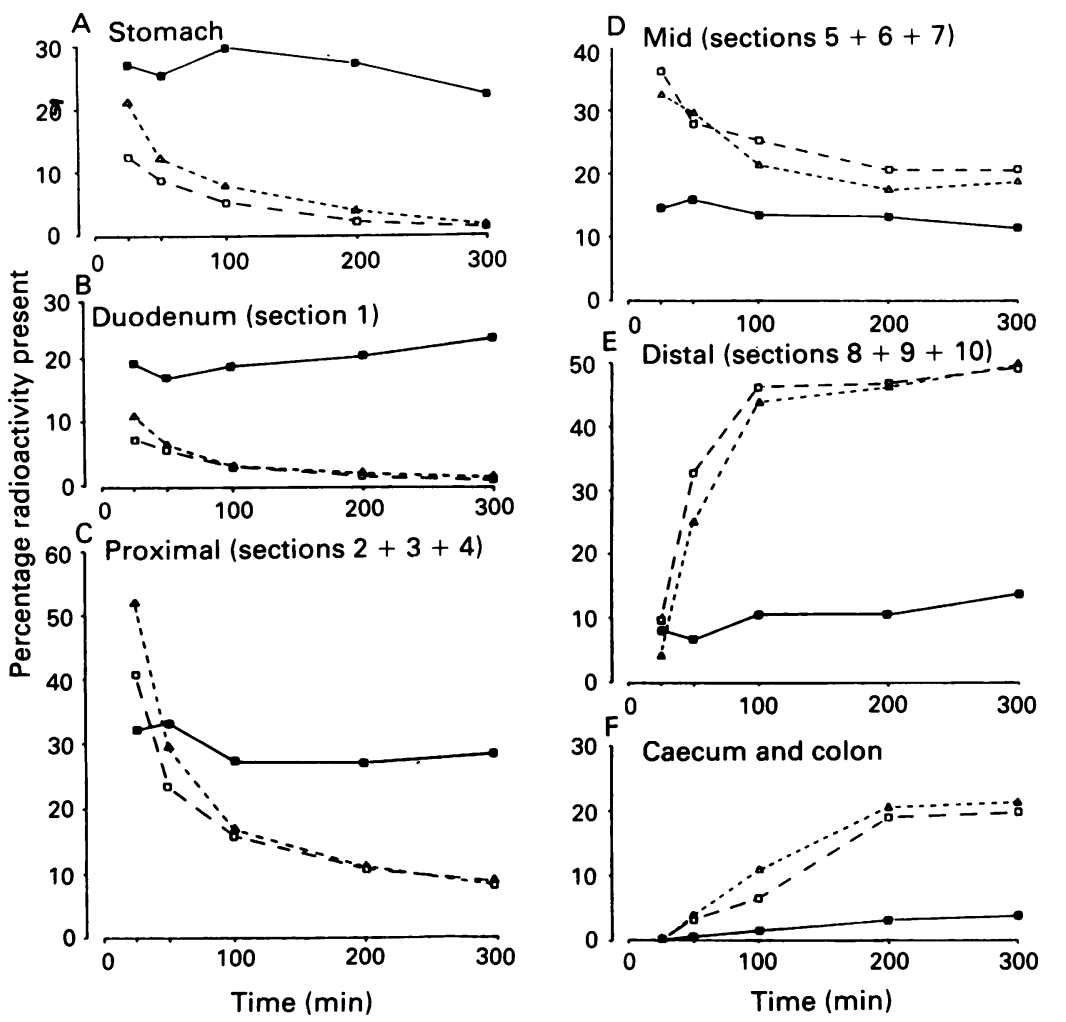

Figure 1: The residence time of radioactive label within each section of the gut; $(A)$ stomach, $(B)$ duodenum, $(C)$ proximal small intestine, $(D)$ mid small intestine, $(E)$ distal small intestine, (F) caecum and colon. Liquid fibre is shown by dark squares/solid lines, sodiumdodecylsulphate (SDS) control by light squares/dashed line, and water control is shown by light triangles and dotted line. Each point represents the mean percentage marker present at each time interval after gavage when the groups of rats were killed. There were five rats in each group except the 50 minute water control in which there were seven and the 100 and 200 minute water controls in which there were six.
Electric, or Model 1201, Pho Camer, Nuclear Chicago, Amsterdam, The Netherlands) and drank $250 \mathrm{ml}$ of the appropriate liquid that had been labelled with $1.85 \mathrm{MBq}{ }^{99 m} \mathrm{Tc}$-tin colloid (Amersham, Bucks, UK). Both drinks were consumed within two minutes. A dorsal image was taken every two minutes for the first 30 minutes and every 10 minutes subsequently. A left lateral image was taken at the end of the study after the ingestion of an additional 1.00 $\mathrm{MBq}{ }^{99 \mathrm{~m}} \mathrm{Tc}$-tin colloid in $200 \mathrm{ml}$ water to correct for tissue attenuation and lateral movement of the label. ${ }^{4}$

The images were stored on a computer and movement of the label out of the stomach was analysed by outlining an area of interest of the stomach region and plotting the percentage of radioactive counts present against time. The values were corrected for movement of the marker away from the dorsal surface by incorporating the data from the lateral image. The time for $10 \%$ of the label to empty (which includes any lag period) and the half emptying time or $t^{1 / 2}$ (time for $50 \%$ to empty) were derived from the graphs. The average rate of emptying was calculated as the slope of emptying between $\mathrm{t} 10 \%$ and $\mathrm{t} 1 \frac{1}{2}$.

\section{STATISTICAL ANALYSIS}

The results are expressed as means with standard errors as appropriate. Statistical significance was established using Wilcoxon's rank sum tests in the rat study and paired Student's $t$ tests in the human study.

\section{Results}

A midline incision was made and the gut ligated in situ at the lower oesophageal sphincter, the pylorus, the ileocaecal sphincter, and the caecocolonic junction. It was carefully dissected out, beginning with the distal small intestine and colon. Great care was taken not to disturb the gut contents or to stretch the gut. It was then transferred into a long trough containing saline at $37^{\circ} \mathrm{C}$. The trough was pulled under a scintillation counter to obtain a profile of the distribution of the radioactivity along the gut. ${ }^{2}$

The radioactivity profiles were divided into 12 sections; stomach, caecum and colon, and 10 sections of the small intestine of equal length. The geometric centre (GC) of the label was calculated for each rat from the formula ${ }^{3}$

$\mathrm{GC}=\Sigma$ (fraction of radioactivity $\times$ section number $)$.

\section{HUMAN STUDY}

Ten healthy male volunteers aged 21 to 31 years (mean 23.6 years) gave written informed consent to the studies, which were passed by the local Ethical Committee. Each volunteer had two studies performed in a random order; a test in which they took liquid fibre $(0.85 \% \mathrm{wt} / \mathrm{vol}$ EHEC), and a control in which they took a placebo drink (Table I). The studies began at 1000 am after an overnight fast from 2000 the previous day. They were instructed to eat the same foods and follow the same routine on the day before both occasions. The volunteers sat in front of a gammacamera (Radicamer II, General
TABLE II Mean values (SEM) for the 10 human volunteers. All values significantly different $(p<0.05)$

\begin{tabular}{lcc}
\hline & Control & Liquid fibre \\
\hline Half emptying time (min) & $17 \cdot 7(2 \cdot 0)$ & $55 \cdot 8(7 \cdot 1)$ \\
$\mathrm{T}_{10 \%}$ (min) & $7 \cdot 0(0 \cdot 8)$ & $19 \cdot 4(2 \cdot 4)$ \\
Rate of emptying $(\% / \mathrm{min})$ & $4 \cdot 49(0 \cdot 62)$ & $1 \cdot 60(0 \cdot 31)$ \\
\hline
\end{tabular}



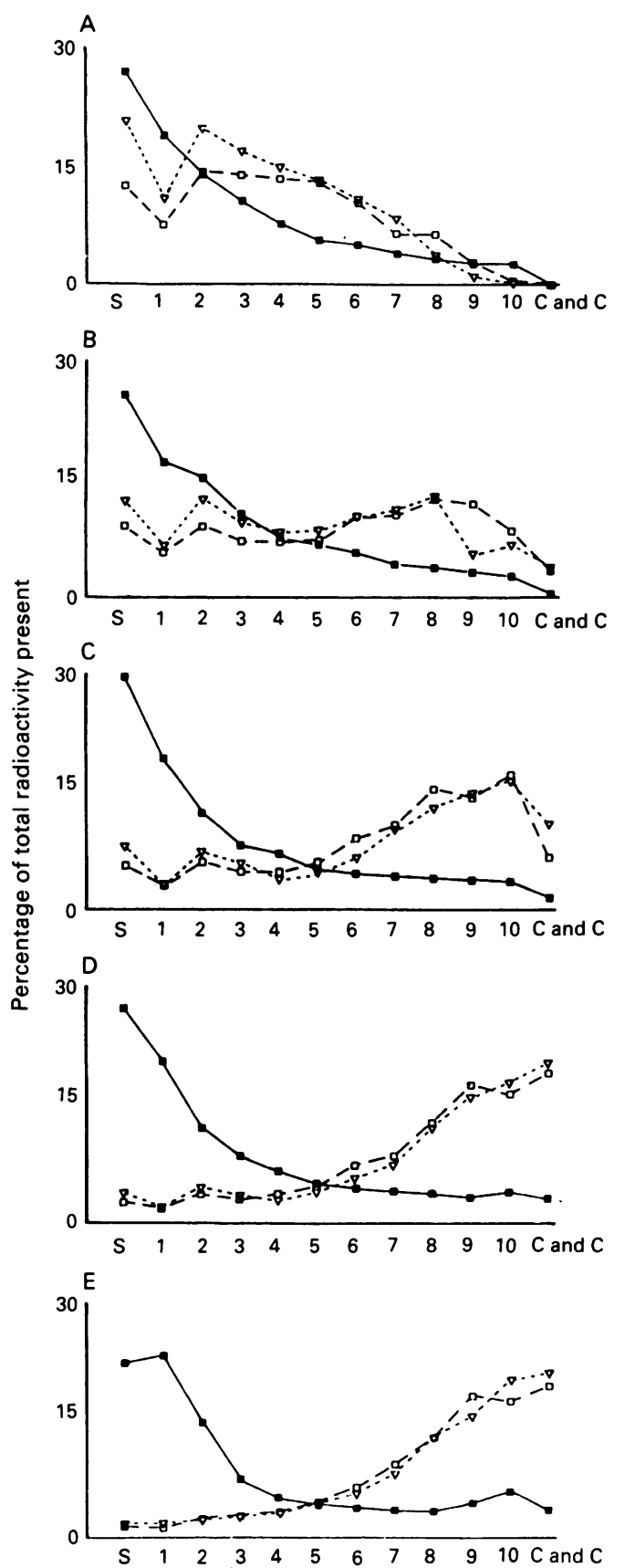

Figure 2: Profiles of the mean radioactivity distributions along the length of the intestine of groups of rats killed at each time interval after gavage; $(A) 25$ minutes; $(B) 50$ minutes, $(C) 100$ minutes, $(D) 200$ minutes, $(E) 300$ minutes. $C$ and $C=$ caecum and colon. $S=$ stomach. Symbols as in Fig 1.

sections (Fig $1 \mathrm{E}$ ) and then entered the caecum and colon more slowly (Fig 1F).

The geometric centres and the average position of the centre of the label also showed a clear retardation of gut movement by the liquid fibre (Fig $3 \mathrm{~A}$ and $\mathrm{B}$ ).

\section{HUMAN STUDY}

The half time for gastric emptying was significantly prolonged in all volunteers from a mean of 17.7 minutes (SEM 2.0) on placebo to 55.8 minutes (SEM 7·1) on liquid fibre (Table II; $\mathrm{p}<0.001)$. This incorporated both the development of a lag time and a decrease in the rate of emptying. The $\mathrm{t}_{10 \%}$ values were statistically prolonged by liquid fibre from 7.0 minutes
(SEM $0 \cdot 8$ ) on the placebo to $19 \cdot 4$ minutes $(2 \cdot 4)$ on liquid fibre (Table II; $p<0.001$ ). The average rate of emptying (taken from the slope between $\mathrm{t}_{10 \%}$ and $\left.\mathrm{t} 1 / 2\right)$ was significantly slower after liquid fibre, being $1.60 \%$ per minute compared with $4.49 \%$ per minute with placebo $(\mathrm{p}<0.01)$. The mean emptying curves (Fig 4) showed that liquid fibre emptied more regularly and more like a solid would empty than the typical exponential decrease in the liquid placebo. There was significantly more radioactivity present in the stomach region with liquid fibre than placebo at all times between 8 and 50 minutes $(p<0.05)$. There was also significantly less liquid fibre in the stomach during the first two minute frame compared with placebo (Fig 4).

\section{Discussion}

The rat study showed that liquid fibre dramatically delayed the delivery of the marker from the stomach into the small intestine and subse-
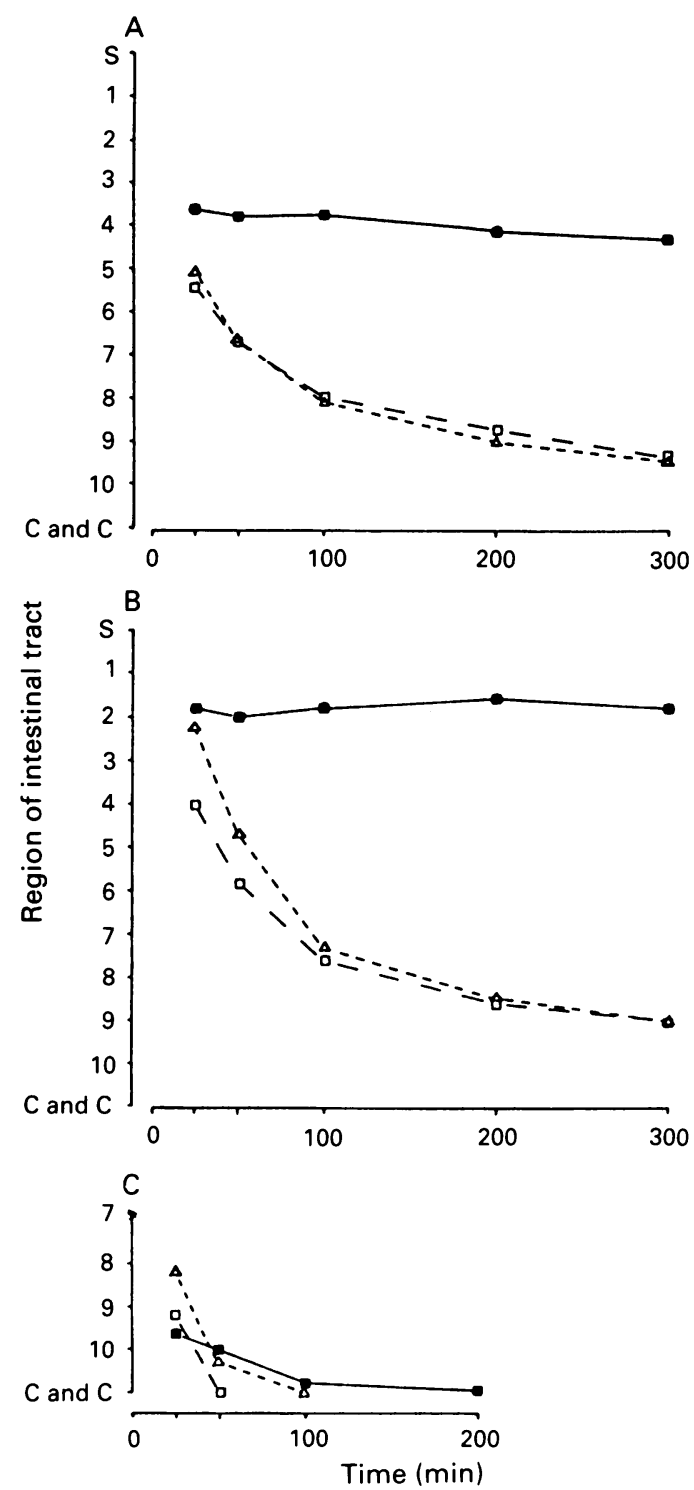

Figure 3: The mean position of $(A)$ the geometric centre of the label, $(B)$ the furthest progression of $50 \%$ of the marker, and (C) the head of the label at each time interval after gavage. Liquid fibre is shown by dark squares/solid lines, SDS control by light squares/dashed line, and water control is shown by light triangles and dotted line. $C$ and $C=$ caecum and colon. Symbols as in Fig 1 . 


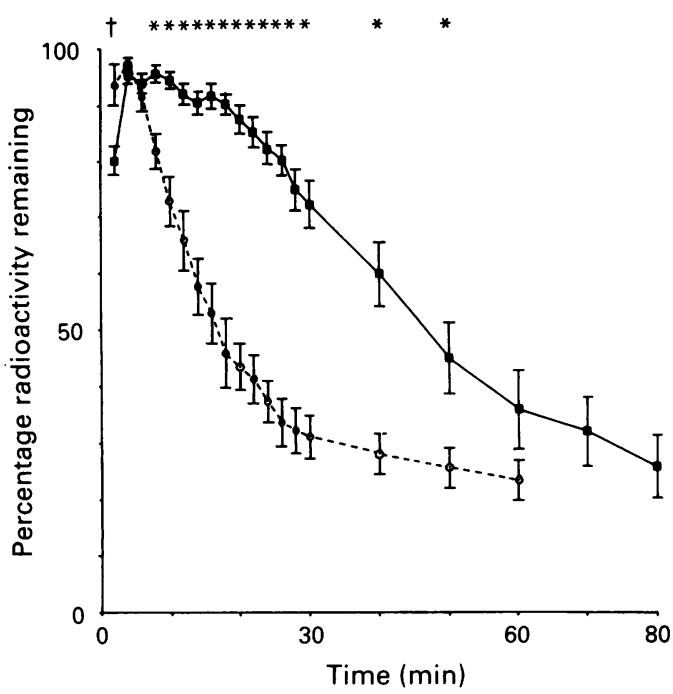

Figure 4: The mean emptying curve for all 10 human volunteers on liquid fibre (dark squares and solid line) and placebo (open circles and dashed line). Each point represents the mean percentage radioactivity present in the stomach region at that time. Standard errors for each of the mean values shown by error bars. Liquid fibre values that are significantly higher than placebo are indicated by an ${ }^{\star}$ above, while the value that is significantly lower is denoted by a + (both $p<0.05$ ).

quently from there into the caecum. Within 25 minutes of the liquids being introduced to the stomach, a distribution was established with most of the marker in the stomach and the remainder spread exponentially down the small intestine. This distribution did not change significantly up until 300 minutes when some marker seemed to have entered the duodenum and there was a slight increase at the distal small intestine. This suggests that the liquid fibre distributed itself as a liquid within 25 minutes, before it had time to reach gelling temperature and that intestinal contents were fairly static for at least 200 minutes; a firm gel was seen in the stomach after 25 minutes.

The results of the human study show that liquid fibre significantly delayed stomach emptying in normal human volunteers. Liquid fibre delayed both the time at which the stomach first began to empty (the lag), and the rate at which it emptied. It gave a profile that was more typical of solid emptying than liquid. This would suggest that gelling had also taken place in the human stomach.

Liquid fibre seemed to delay transit time through the mouth and oesophagus as significantly less liquid fibre was present in the stomach after two minutes than placebo drink (Fig 4). This could be as a result of gelling in these areas or because of its bioadhesive properties. There was no recorded delay in oesophageal transit with other bioadhesives such as polycarbophil and carbopol ( $250 \mathrm{mg}$ ), presumably because the polymers were given within gelatin capsules. ${ }^{5}$ These other bioadhesive substances have only produced a slight but non-significant delay in stomach emptying in man. ${ }^{5}$

Some soluble non-starch polysaccharides (also called 'dietary fibres') that form viscous solutions have been seen to have a similar but less pronounced delaying effect on gastric emptying. For example, $15 \mathrm{~g}$ pectin delayed the half emptying time of an egg sandwich meal to 116 minutes (compared with 71 minutes with $15 \mathrm{~g}$ of the less viscous methyl cellulose), ${ }^{6}$ and when $1 \%$ guar gum was incorporated in an $865 \mathrm{~kJ}$ milkshake, the 'mean gastric transit time' of obese volunteers increased from 69 to 112 minutes.

Viscous polysaccharides have been shown to be useful at controlling hyperglycaemia ${ }^{8}$ and reducing the rapid absorption of nutrients in patients with dumping syndrome. ${ }^{9}$ This is probably because of both their viscous nature and their effect on gastric emptying. In addition, some viscous polysaccharides have been shown to be efficient at decreasing energy intake and causing weight loss in obese subjects; in the same experiments referred to above, $15 \mathrm{~g}$ pectin increased satiety two hours after a meal and increased the time to the next meal from 6 to 10.6 hours ${ }^{6}$ while 2 g guar gum significantly lengthened the time to maximum hunger. ${ }^{7}$ The precise mode of action has not been definitely established but their simultaneous slowing of gastric emptying infers that this mechanism is involved. For this reason liquid fibre would be expected to reduce the rate of nutrient absorption and possibly to inhibit hunger signals in man because of the distension of the stomach for an extended period of time. Volunteer studies will follow to measure the effects of liquid fibre on the absorption of glucose, insulin responses, and the effects of blood cholesterol as well as hunger ratings and food intake.

Products on the market at the moment that contain viscous polysaccharides are not very palatable and may be rather difficult to take over the long periods of time that may be necessary for controlling diabetes, dumping syndrome or the correction of obesity. The liquid form of the substance tested allows greater patient acceptability, which in turn may improve longterm compliance. Some of the current products are in tablet form, and this leads to the danger of intestinal obstruction or rupture caused by the hydrophilic swelling of these products in the gastrointestinal tract, particularly in the oesophagus. ${ }^{1011}$ As liquid fibre is already fully hydrated when it is consumed, the danger of obstruction occurring in the gut is dramatically reduced.

In conclusion, the large and significant delay in gastric emptying seen in these studies supports the idea that liquid fibre may be useful clinically. It may be useful in the management of dumping syndrome in which symptoms are thought to be caused by an unnaturally rapid delivery of the meal out of the stomach into the intestine. It may help control diabetes by delaying and slowing the rate of absorption of glucose although delayed gastric emptying may prove to be a problem in these patients who often have slow emptying as a feature of the disease. It is possible that these beneficial clinical effects could be influenced by the fate of the compound in the colon. Little is known of this, but 10 days of feeding liquid fibre at a slightly lower dose of $200 \mathrm{ml} / \mathrm{d}$ had no significant effect on stool mass but slightly reduced transit time compared with placebo suggesting mild laxative properties (unpublished data). Liquid fibre could also be useful as an aid to weight reduction but further 
studies are needed in which eating behaviour is measured directly.

This work was supported by funding from Kabi Invent AB.

1 Trowell H. Definition of dietary fibre and hypotheses that it is a protective factor in certain diseases. Am $\mathcal{F}$ Clin Nutr 1976; 417-27.

2 Brown NJ, Worlding J, Rumsey RDE, Read NW. The effect of guar gum on the distribution of a radiolabelled meal in the gastrointestinal tract of the rat. Br F Nutr 1988; 59:223-31.

3 Miller MS, Galligan JJ, Burks TF. Accurate measurement of intestinal transit in the rat. $\mathcal{F}$ Pharmacol Methods 1981; 6 : 211-7

4 Collins PJ, Horowitz M, Shearman DJC, Chatterton BE. Correction of tissue attenuation in radionuclide gastric emptying studies: a comparison of a lateral image method and a geometric mean method. Br 7 Radiol 1984; 57: $689-95$
5 Harris D, Fell JT, Sharma H, Taylor DC, Li J. Studies on potential bioadhesive systems for oral drug delivery. STP Pharma 1989; 5: 852-6.

6 DiLorenzo C, Williams CM, Hainal F, Valenzuela JE. Pectin delays gastric emptying and increases satiety in obese subjects. Gastroenterology 1988; 95: 1211-5.

7 Wilmhurst P, Crawley JCW. The measurement of gastric transit time in obese subjects using "Na, and the effects of energy content and guar gum on gastric emptying and satiety. Br f Nutr 1980; 44: 1-6.

8 Jenkins DJA, Leeds AR, Gassull MA, Cochet B, Alberti KGMM. Decrease in postprandial insulin and glucose KGMM. Decrease in postprandial insulin and glucose 20-3.

9 Leeds AR, Ralphs DNL, Ebied F, Metz G, Dilawari JB Pectin and the dumping syndrome: Reduction of symptoms and plasma volume changes. Lancet 1981; i: 1075-8.

10 Morse JMD, Malloy WX. Oesophageal obstruction caused by Cal-ban. Gastroenterology 1990; 98: 805.

11 Gebhard RL, Albrecht J. The diet pill that worked. $N$ Engl $f$ Med 1990; 322: 702 . 